\title{
Northern Hemisphere observations of ICRF sources on the USNO stellar catalogue frame
}

\author{
A. Fienga ${ }^{1}$ and A. H. Andrei ${ }^{2}$ \\ ${ }^{1}$ Institut de Mécanique Céleste et de Calcul des Éphémérides (IMCCE) - Observatoire de Paris, UMR 8028 du CNRS, \\ 77 avenue Denfert-Rochereau, 75014 Paris, France \\ 2 Grupo de Estudos em Astronomia-OV/UFRJ and Observatório Nacional/MCT, R. Gal. José Cristino 77, S. Cristovão, \\ Rio de Janeiro, Brasil
}

Received 21 July 2003 / Accepted 4 November 2003

\begin{abstract}
The most recent USNO stellar catalogue, the USNO B1.0 (Monet et al. 2003), provides positions for 1042618261 objects, with a published astrometric accuracy of 200 mas and five-band magnitudes with a 0.3 mag accuracy. Its completeness is believed to be up to magnitude 21th in $V$-band. Such a catalogue would be a very good tool for astrometric reduction. This work investigates the accuracy of the USNO B1.0 link to ICRF and give an estimation of its internal and external accuracies by comparison with different catalogues, and by computation of ICRF sources using USNO B1.0 star positions.
\end{abstract}

Key words. astrometry - reference system

\section{Introduction}

Like its predecessor, the USNO A2.0 catalogue (Monet et al. 1998), the USNO B1.0 (Monet et al. 2003) was compiled from the digitisation of photographic sky survey plates by the USNO PPM. It furnishes positions and magnitudes for 1042618261 objects and proper motions for a part of the catalogue. Thanks to its star density, the USNO B1.0 catalogue provides all-sky coverage and completeness down to 21 th mag in the $V$ band. The published astrometric accuracy at J2000 is about 200 mas, and about $0.3 \mathrm{mag}$ for the five color photometry.

The USNO B1.0 is the latest of the USNO catalogues (UJ1.0, USNO A2.0, USNO A1.0) covering both sky hemispheres on different dates and magnitudes. For this version special care was taken in the link to the Tycho-2 frame, to which the catalogue orientation ultimately refers. An attempt was also made to estimate proper motions between the two or three epochs available per star identification. The magnitude determination and the separation between stellar and extended objects (mainly galaxies) are also important features of the catalogue. The photometry, however, is not within the scope of the present work, which concentrates rather on the astrometric characteristics.

To avoid the use of saturated star images in the link process between the Tycho- 2 and the USNO B1.0, a secondary plate survey was needed. The latter, based on the scan of proper motion plate surveys, had a limiting magnitude dimmer than that of the Tycho- 2 catalogue. It was then possible to use Tycho- 2 as

Send offprint requests to: A. Fienga, e-mail: fienga@imcce. fr a secondary frame for the link between the USNO B1.0 catalogue and the Tycho- 2 catalogue. Yet, as stressed in the catalogue presentation (Monet et al. 2003), the accuracy and completeness for brighter stars is lower than for fainter stars. Here, the astrometry of optical counterparts of ICRF sources and comparisons to different catalogues will be used to investigate the variation of precision with magnitude.

The external variation of precision, as consequence of zonal errors, is accessed in the B1.0 catalogue publication (Monet et al. 2003) by comparison to the SDSS Early Data Release, EDR (Stoughton et al. 2002). The SDSS project will map onequarter of the entire sky and perform a redshift survey of galaxies, quasars and stars. The SDSS EDR covers $450 \mathrm{deg}^{2}$, providing positions, proper motions, photometry and spectroscopy. Its estimated astrometry is better than 100 mas. Within the limited coverage of the SDSS EDR, the comparison indicates B1.0 zone errors to about 250 mas, with a mean dispersion of 130 mas, for stars of magnitude between 17th and 19th magnitude in the SSDS $g^{*}$ band. In the present work, the astrometry of ICRF sources will be used to discuss the characteristics of such zonal errors. The GSC 2.2 catalogue (STScI 2001) and the M 2000 catalogue (Rapaport et al. 2001) will be used. In April 2002, the GSC 2.2 catalogue numbered 455851237 objects, up to magnitude 18.5 in $F$ and 19.5 in $J$. In a previous work (Fienga \& Andrei 2002, hereafter Paper I), the internal accuracy of this preliminary version of GSC II was determined to be about 200 mas. The next version of the GSCII catalogue will include proper motions and is expected to be released in early 2004. Unlike the GSC and USNO catalogues, the M 2000 is based on meridien drift scannings of a restricted part of the 
Northern Hemisphere (between +11 and 18 degrees in declination). This catalogue contains 2300000 stars down to the 16 th mag with a mean astrometric accuracy better than 50 mas.

For the astrometry of solar system objects or other types of dynamically interesting objects, and in general for small field astrometric reductions referred to the ICRS, the USNO B1.0 catalogue would be a tool of choice. Hence, it is interesting and important for the potential users of the USNO B1.0 catalogue to investigate the accuracy of the link between this catalogue and the ICRF.

In the next sections of the paper we give the essentials of the observation made, and of the method used to determine the external accuracy of stellar catalogues. In Sect. 3, the comparisons to the ICRF are presented. In Sect. 4, the comparisons are towards brighter frames. The summary of the results and conclusions is presented in the final section.

\section{Observations of optical counterparts of ICRF sources}

\subsection{Method}

All results have been obtained from observations of optical counterparts of ICRF sources performed in 2001 at the Observatoire de Haute-Provence. The complete description of the observations and the reduction process used to compute observed positions of the ICRF sources is given in Paper I. Here the elements relevant for the present investigation are briefly reviewed. The observations were obtained with the $1.20 \mathrm{~m}$ telescope. The CCD is 1024 by 1024 pixel and the scale is 0.68 arsec per pixel. The field of view is then about 144 squared arcmin. Each source was observed three times with the Cousins $V$ and $R$ filters (540 and $610 \mathrm{~nm}$ ). The flatfielding and the bias corrections were made using the CCD reduction package ECLIPSE 4.2 (2002). The extraction of the sources was realized with an automatic extraction procedure (Alard 2002). For the astrometric reduction process, the software PRIAM was used (Fienga 1999; Fienga et al. 2002). It includes computation of apparent coordinates (corrections of annual and diurnal aberrations, deflection of light), corrections of refraction by mapping functions handling the meteorological conditions and the wavelengths of observation. The resulting positions are then regarded to be cleaned of important uncertainties otherwise induced by the reduction process.

The objects were observed from $23 \mathrm{~h}$ to $13 \mathrm{~h}$ in right ascension and from 0 degree to 45 degrees in declination (though mostly between +5 and +20 degrees). The visual magnitudes of the stars used in the reduction process (see Fig. 1) are fainter than the 11th magnitude as the magnitudes of the extragalactic sources. To be able to test the quality of the link between the USNO B1.0 and ICRF, we chose the USNO B1.0 catalogue as reference stars for the reduction process. Figure 1 shows histograms and cumulative histograms of the distributions of the $R$-magnitudes of USNO B1.0 and GSC 2.2 stars used in the reduction. This distribution of the USNO B1.0 magnitudes is the same as the one published in Paper I for the USNO A2.0 catalogue. It can be explained because the limiting magnitude of detection for these observations was already achieved with the
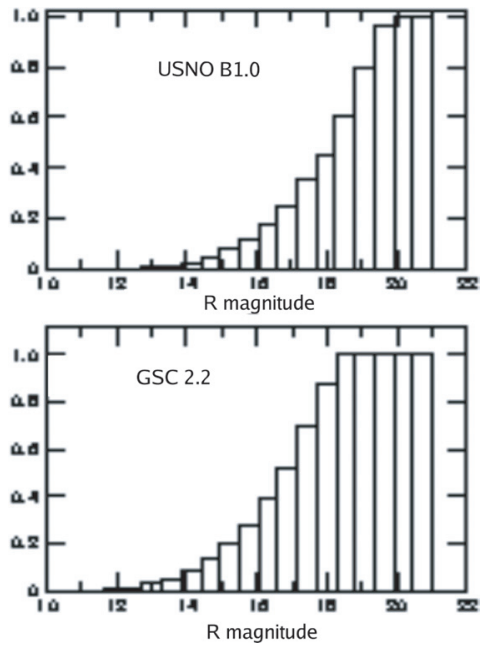

Fig. 1. Cumulative histograms of the $R$ magnitudes of USNO B1.0 and GSC 2.2 stars used for the astrometric reduction of the optical counterparts.

USNO A2.0 stars due to the quality of the observations (seeing about 4 arcsec, time of exposure about $3 \mathrm{~min}$ ). This fact allows us to compare directly the quality of these two catalogues in the same range of magnitudes. The mean number of stars per field is also the same for the USNO B1.0 catalogue and the USNO A2.0 catalogue, about 90 stars per field. The mean number of GSC 2.2 stars is about 60 stars per observed field.

\subsection{Comparisons of deduced positions to ICRF}

In order to obtain the zonal departures from the ICRF, the astrometric reduction of optical counterparts using B1.0 reference stars is employed. The method has the further advantage, compared to direct catalogue-to-catalogue comparison, of providing a swept on magnitude, making the results valid for the average of a large magnitude profile in the catalogue. Any true difference between the optical and radio positions must be much smaller than the other sources of errors, and would not be relevant for the present study.

Figure 2 presents the differences obtained after comparisons between ICRF positions and positions of extragalactic sources deduced from reductions using USNO B1.0 stars. Systematic effects are present in right ascension and declination. In declination, a systematic offset of about +170 mas could be estimated over all the regions under study. Two distinct groups appear on the $\Delta \alpha \cos \delta$ distribution versus right ascension. The two groups can be taken as contained in the intervals $\left[23^{\mathrm{h}}, 6^{\mathrm{h}}\right]$ and $\left[6^{\mathrm{h}}, 13^{\mathrm{h}}\right]$. The respective averages are also shown in Cols. 3 and 4 of Table 1. A linear fit describes the two groups of right ascension zonal departures at the $3 \sigma$ level. The angular coefficient is $(+13.72 \pm 4.10) \mathrm{mas} / \mathrm{h}$. The variance of the adjustment is smaller than that of the distribution, and the variances of residuals from the linear fit are slightly smaller than those for the distributions within each group. Both the existence of two distinct groups and the good linear fit support the understanding of the B1.0 as a catalogue of reliable internal coherency, although containing important zonal errors. 

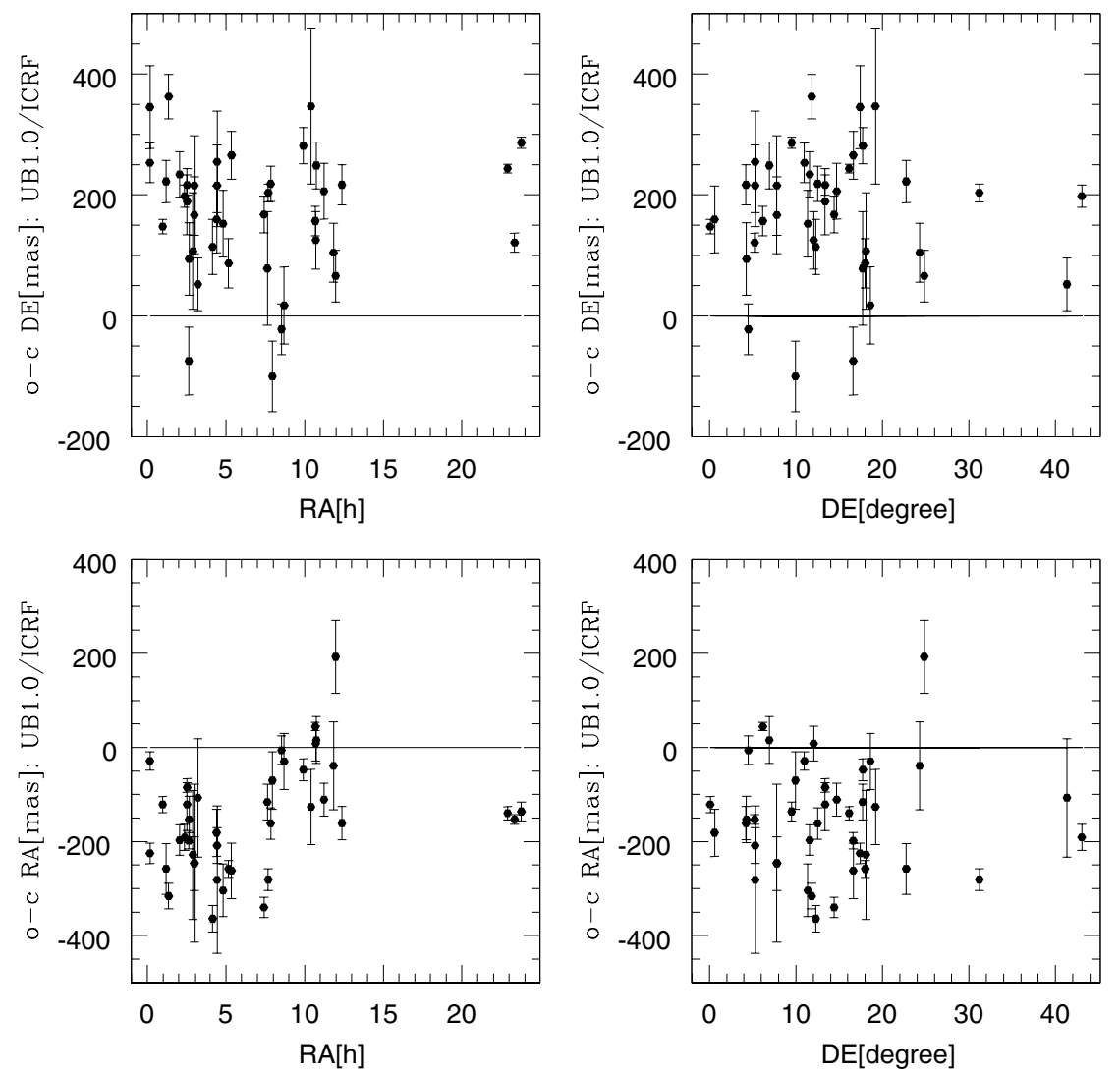

Fig. 2. Normal points of observed positions of ICRF optical counterparts computed with USNO B1.0 stars. The error bars are the standard errors of the observed positions minus the ICRF positions.

Table 1. Means and standard deviations, in mas, of the observed minus ICRF right ascension and declination of the 38 ICRF sources observed in optical wavelengths and reduced using the USNO A2.0 catalog (Cols. 3 and 4), and the preliminary version of the GSC II catalog (Cols. 5 and 6), the USNO B1.0 catalogue (Cols. 7 and 8). Columns 9 and 10 give the results for mean direct differences between USNO B1.0 positions and M 2000 positions for common stars in 17 observed fields.

\begin{tabular}{|c|c|c|c|c|c|c|c|c|c|}
\hline \multirow[b]{2}{*}{$\alpha$ Zones } & \multirow[t]{2}{*}{ ICRF } & \multicolumn{2}{|c|}{ 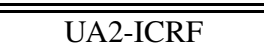 } & \multicolumn{2}{|c|}{ "GSC 2.2-ICRF } & \multicolumn{2}{|c|}{ "UB1-ICRF } & \multicolumn{2}{|c|}{ UB1-M 2000} \\
\hline & & $\begin{array}{r}\Delta \alpha \cos \delta \\
{[\mathrm{mas}]}\end{array}$ & $\begin{array}{r}\Delta \delta \\
{[\mathrm{mas}]}\end{array}$ & $\begin{array}{r}\Delta \alpha \cos \delta \\
{[\mathrm{mas}]}\end{array}$ & $\begin{array}{r}\Delta \delta \\
{[\mathrm{mas}]}\end{array}$ & $\begin{array}{r}\Delta \alpha \cos \delta \\
{[\mathrm{mas}]}\end{array}$ & $\begin{array}{r}\Delta \delta \\
{[\mathrm{mas}]}\end{array}$ & $\begin{array}{r}\Delta \alpha \cos \delta \\
{[\mathrm{mas}]}\end{array}$ & $\begin{array}{r}\Delta \delta \\
{[\mathrm{mas}]}\end{array}$ \\
\hline$[23 \mathrm{~h}, 13 \mathrm{~h}]$ & 38 & $30_{ \pm 173}$ & $254_{ \pm 153}$ & $57_{ \pm 131}$ & $59_{ \pm 138}$ & $-152_{ \pm 116}$ & $169_{ \pm 104}$ & $-110_{ \pm 92}$ & $146_{ \pm 61}$ \\
\hline$[23 \mathrm{~h}, 6 \mathrm{~h}]$ & 22 & $-55_{ \pm 112}$ & $269_{ \pm 185}$ & $13_{ \pm 132}$ & $104_{ \pm 124}$ & $-208_{ \pm 81}$ & $181_{ \pm 97}$ & $-148_{ \pm 77}$ & $152_{ \pm 66}$ \\
\hline$[6 \mathrm{~h}, 13 \mathrm{~h}]$ & 16 & $136_{ \pm 158}$ & $255_{ \pm 180}$ & $111_{ \pm 104}$ & $24_{ \pm 137}$ & $-87_{ \pm 118}$ & $156_{ \pm 114}$ & $-40_{ \pm 79}$ & $136_{ \pm 55}$ \\
\hline
\end{tabular}

In Table 1, one can also find the means and standard deviations estimated for the differences in positions of ICRF optical counterparts estimated with the GSC 2.2 and the USNO A2.0 catalogues. The zonal departures found with the USNO B1.0 reduction are similar to those obtained in Paper I with the GSC 2.2 stars and USNO A2.0 stars. The effects in right ascension for the B1.0 catalogue are also present for the A2.0 and for the GSC 2.2 catalogues. Furthermore, as the GSC 2.2 catalogue is much closer to the ICRF than the A2.0, its comparison to the B1.0 is particularly interesting. Figure 3 presents the differences between ICRF optical counterpart positions obtained using the reference stars from the GSC 2.2 in the reduction process and ICRF positions. Even if the systematic effects are less important than those detected in the USNO B1.0, a similar trend in right ascension is noticed before and after $6 \mathrm{~h}$.

These catalogues are all based on scanning of mostly identical photographic plates. One hypothesis that could explain such an error in right ascension could be a mislinkage between overlapping plates done before and after $6 \mathrm{~h}$ in right ascension.

Tests were also made using the proper motions given for the USNO B1.0 stars. In the observed fields, $25 \%$ of the USNO B1.0 stars have non-zero proper motions. Only 11 fields have enough stars with proper motions for an astrometric reduction. After reducing positions of ICRF sources using USNO B1.0 stars with and without proper motions, it seems that the USNO B1.0 field of proper motions, in the observed regions, is quite reliable. 

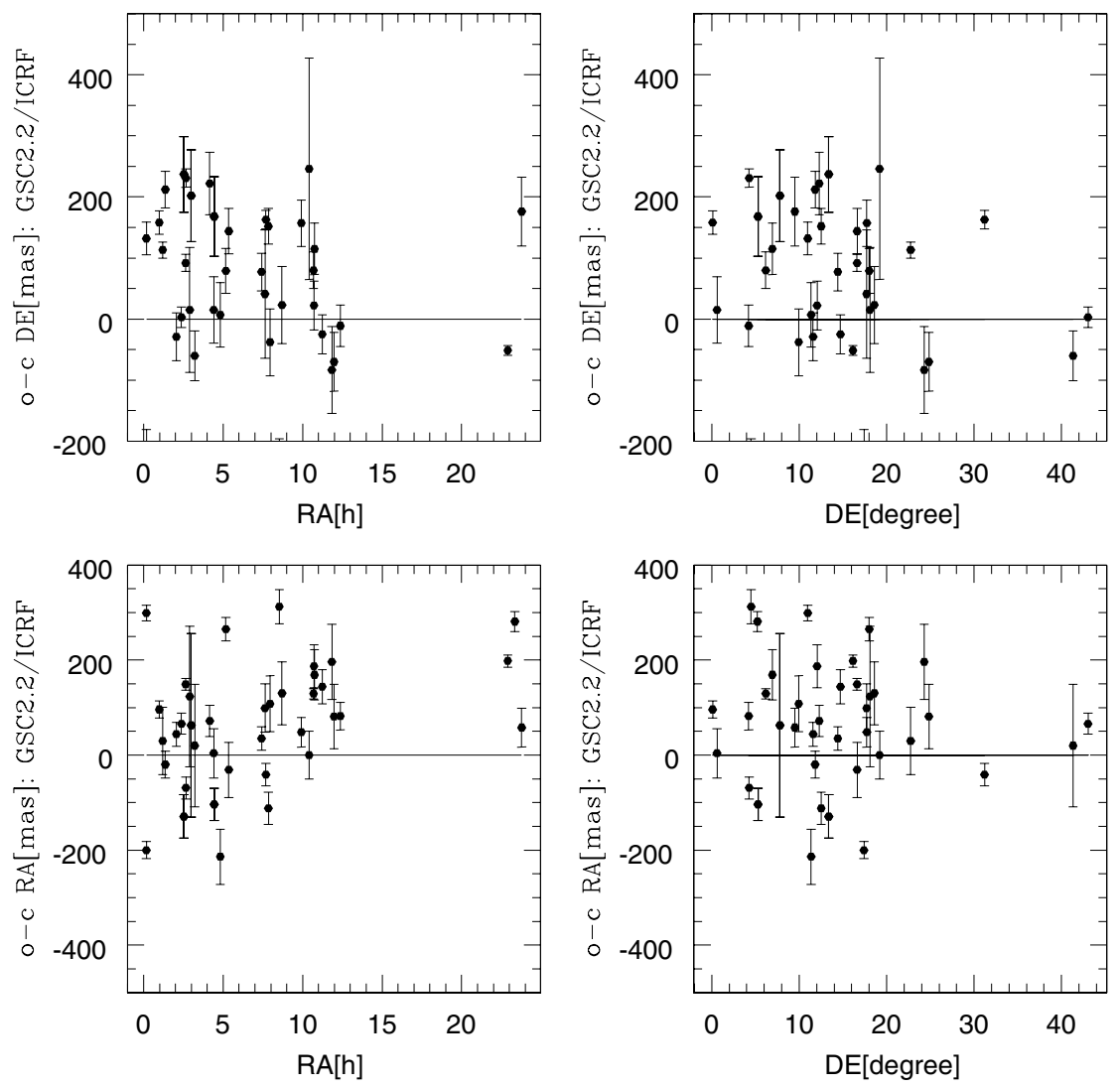

Fig. 3. Normal points of observed positions of ICRF optical counterparts computed with GSC 2.2 stars. The error bars are the standard errors of the observed positions minus the ICRF positions.

Table 2. Main characteristics of post-reduction residual distributions for the USNO B1.0, USNO A2.0 and GSC 2.2 catalogues. Columns 2 and 3 give the mean number of stars per fields and the mean magnitudes of stars respectively. Columns 4 and 5 give the dispersions of the distributions.

\begin{tabular}{rrrrr}
\hline \hline & $\langle N\rangle /$ field & $\left\langle M_{v}\right\rangle$ & $\sigma[\Delta \alpha \cos \delta]$ & $\sigma[\Delta \delta]$ \\
& & & mas & mas \\
\hline UA2.0 & 90 & 18 & 290 & 260 \\
UB1.0 & 90 & 18 & 190 & 170 \\
GSC 2.2 & 60 & 17 & 180 & 160 \\
\hline
\end{tabular}

\subsection{Post-reduction residuals}

A source of information about the USNO B1.0 astrometry quality is the analysis of the differences between USNO B1.0 positions of stars used in the reduction process and positions of these stars after the reduction process. These differences represent the internal accuracy of the star catalogue, after the fit of a linear model correcting the observational function of errors. Such analysis was done for the USNO B1.0 catalogue. The distributions of the residuals corresponding to this catalogue and for the USNO A2.0 and GSC 2.2 catalogues, extracted from the Paper I, are presented in Fig. 4, and the main characteristics of the distributions are given in Table 2.

On the basis of the standard deviations presented in Table 2, the USNO B1.0 catalogue presents an internal accuracy, about
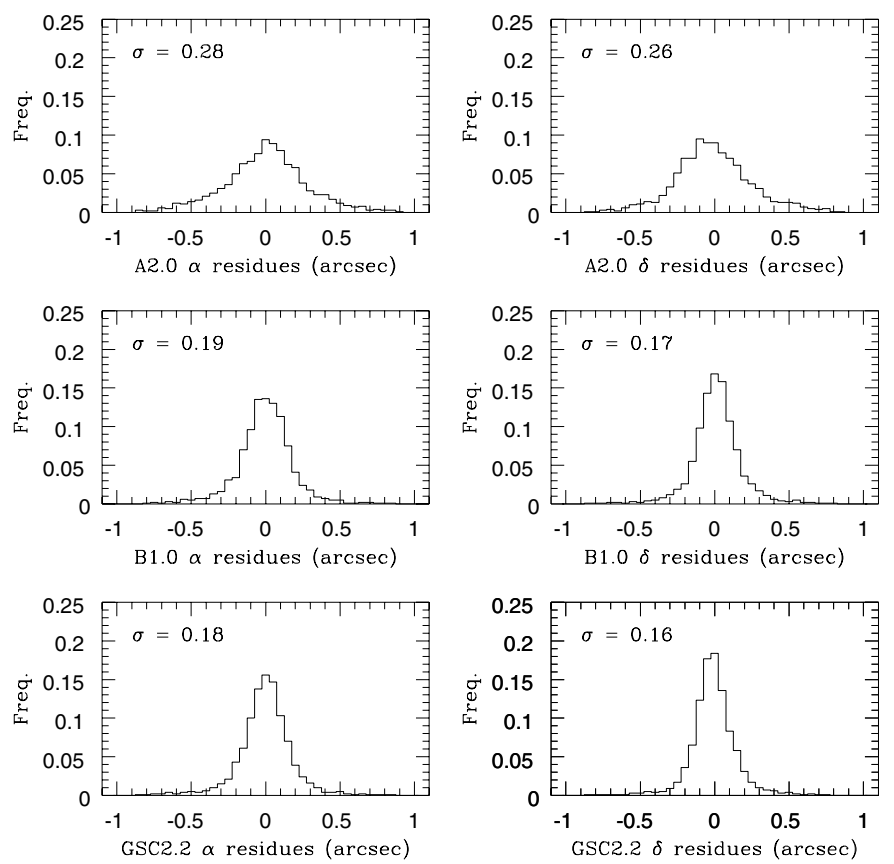

Fig. 4. Post-reduction residuals.

180 mas, comparable to the GSC 2.2 accuracy, about 170 mas. The USNO A2.0 catalogue dispersion is about 280 mas. All these figures are comparable to values already published in Paper I and in Assafin et al. (2001). 

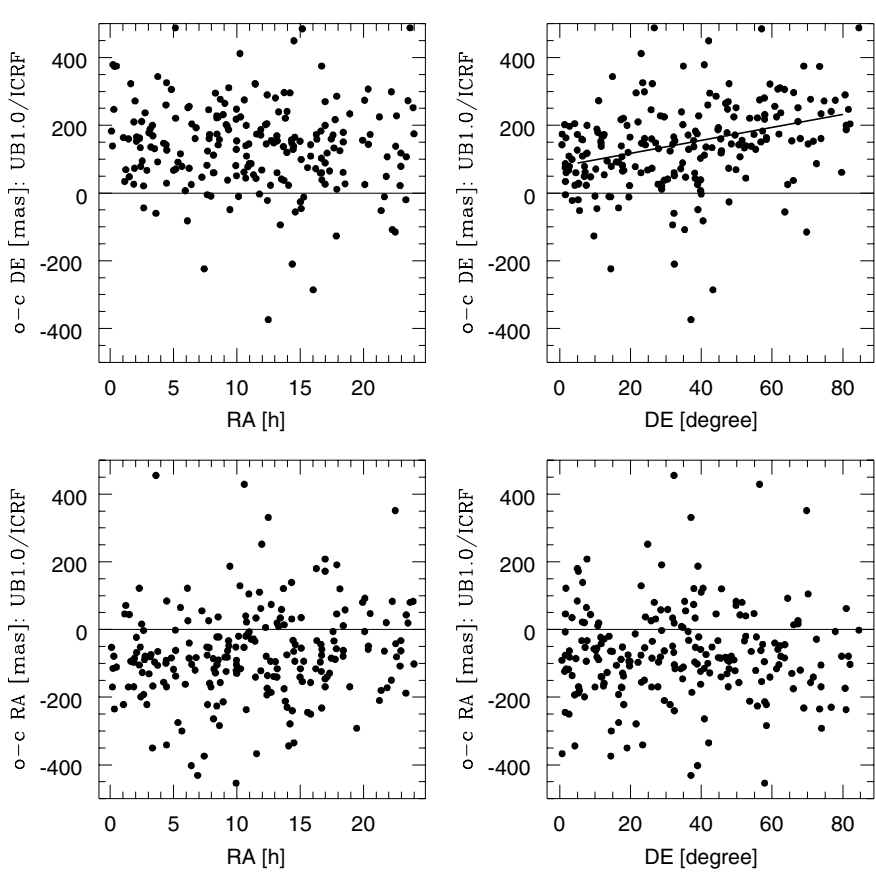

Fig. 5. Comparisons between USNO B1.0 optical positions and ICRF radio positions for the northern hemisphere.

\section{Catalogue-to-catalogue comparison}

\subsection{USNO B1.0 vs. ICRF}

For the Northern Hemisphere and from the B1.0 catalogue, 217 identifications of ICRF sources were found. The 217 obtained differences between B1.0 positions and ICRF positions are presented in Fig. 5. The identification criteria was that the B1.0 candidate would not have proper motions, and that its radial distance from the radio position is smaller than 1 arcsec.

Due to the limited quantity of points, the plots do not enable us to obtain zonal errors. Similarly, due to the generally high magnitudes, such direct comparison is, in principle, limited to the faintest end of the B1.0 catalogue. Systematic offsets as large as 200 mas in right ascension and in declination can be detected. The average B1.0 minus ICRF offsets are $\Delta \alpha \cos \delta=$ $-69 \pm 11$ mas $(\sigma=156$ mas $)$ and $\Delta \delta=+133 \pm 11$ mas $(\sigma=165$ mas). A statistically significant linear trend can be estimated in the distribution in declination of declination residuals. Its angular coefficient is equal to $1.93 \pm 0.32 \mathrm{mas} /$ degree.

The zonal errors previously detected can tested by taking the direct comparison between positions of B1.0 identifications of ICRF sources and ICRF positions, for the observed regions. In this case, due to the very reduced number of sampled points, the identification criteria were tightened, and positive matches were recognised only for B1.0 objects without assigned proper motions, fainter than magnitude 13 , and closer than 600 mas to the radio position, both in right ascension and declination. Enforcing these criteria, 29 sure identifications were retained. The direct optical minus radio differences are plotted in Fig. 6. As seen from the $\Delta \alpha \cos \delta$ distribution versus right ascension plot, the two right ascension groups reappear. Also the linear tendency reappears, with an angular coefficient
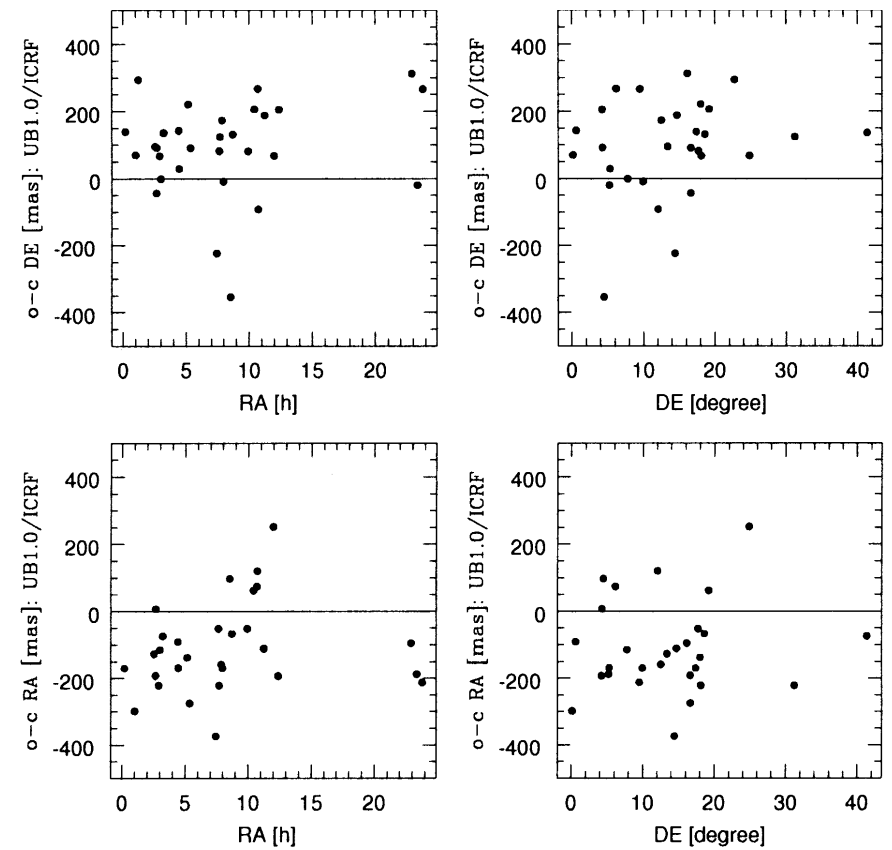

Fig. 6. Comparisons between USNO B1.0 optical positions and ICRF radio positions for the northern hemisphere for observed regions.

of $+15.49 \pm 5.64 \mathrm{mas} / \mathrm{h}$, which agrees with the formerly found coefficient (Sect. 2.2).

\subsection{USNO B1.0 vs. M 2000}

To verify the hypothesis of systematic trends in photographic plate-based catalogues, we have compared directly the positions of the USNO B1.0 stars with positions extracted from a catalogue that is not based on photographic plates. We have chosen the Meridien 2000 (M 2000) catalogue (Rapaport et al. 2001) which is based on the compilation of meridian drift scanning observations taken at the Bordeaux Meridian Circle. The survey is limited to +11 degrees to +18 degrees in declination, but is very dense in this zone (about 2300000 stars down to the magnitude limit $V M=16.3$ ). The mean astrometric accuracy of this catalogue is estimated to be about 5 mas in the magnitude range $11<V M<15$, which degrades to about 50 mas when the faintest stars are considered. For the zone covered by M 2000, we have compared directly the positions given in the USNO B1.0 and the positions of common stars in M 2000. For 17 fields covered by USNO B1.0 and M 2000, we obtained an average number of common stars of 50 .

The obtained mean differences are plotted in Fig. 7, and the means and standard deviations of the mean differences between USNO B1.0 and M 2000 positions are presented in the last columns of Table 1. The right ascension and declination overall offsets previously detected in the ICRF comparisons have seen. In particular, the two right ascension zones of the $\Delta \alpha \cos \delta$ residuals are present. Also the linear fit of right ascension is significant. When adjusted on a star-by-star basis, the angular coefficient is $(+9.32 \pm 1.34)$ mas/h. Taking the average of the fields, the angular coefficient is $(+10.80 \pm 6.31) \mathrm{mas} / \mathrm{h}$. 

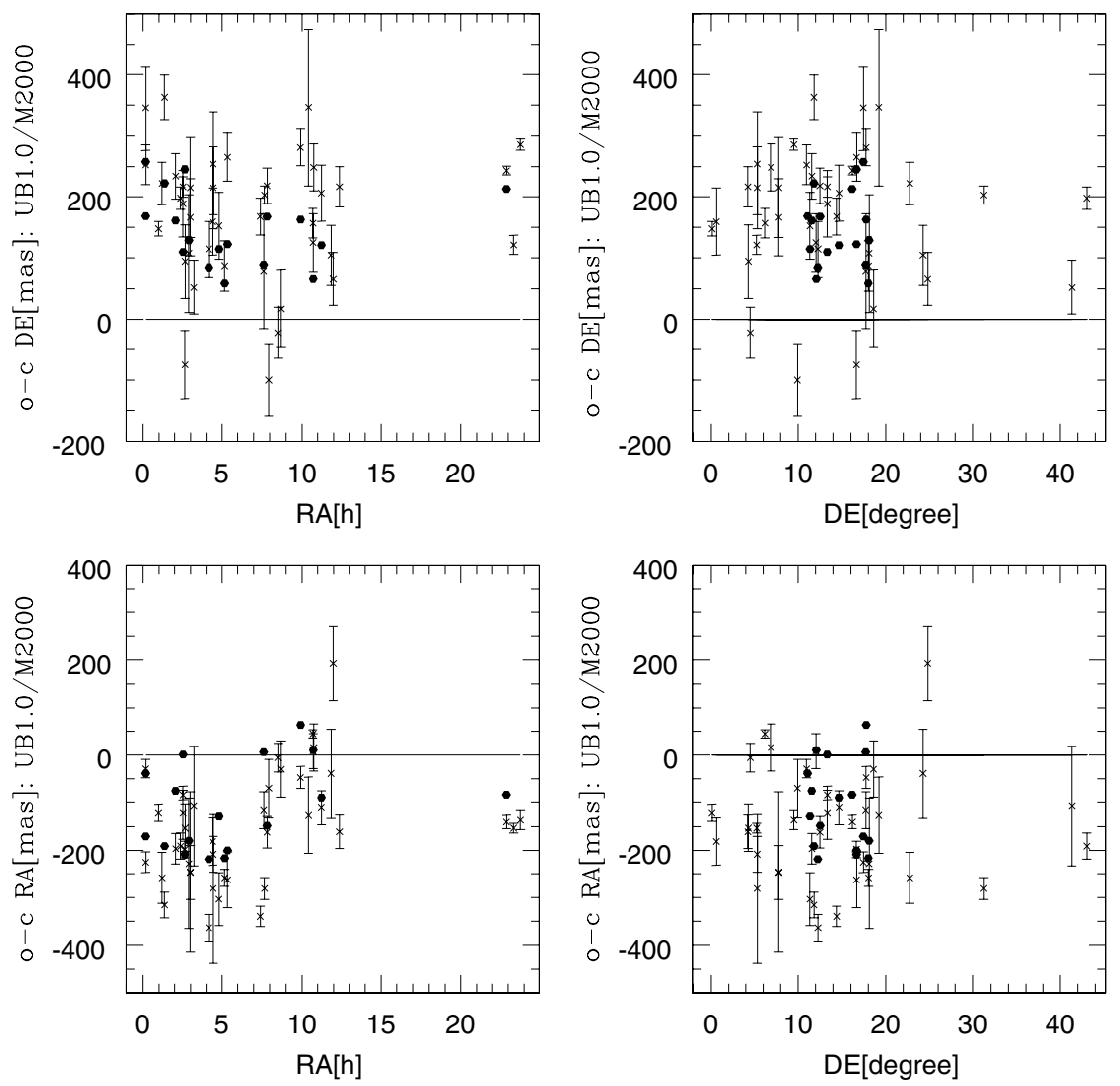

Fig. 7. Comparison between USNO B1.0 and M 2000 positions. In the figure are overplotted i) with black diagonal and errors bars, the normal points of observed positions of ICRF optical counterparts computed with USNO B1.0 stars. The error bars are the standard errors of the observed positions minus the ICRF positions ii) with black hexagons, normal differences for common stars between the USNO B1.0 and M 2000 positions.

In either case, they are in full agreement with the values found for the other magnitude regimens.

As the direct comparison between B1.0 positions and ICRF and M 2000 positions shows results equivalent to those from the present astrometric observations and reductions, these are ruled out as the source of the departures. At the same time, such agreement rejects explanations based on magnitude equations.

Furthermore, as the trends were also detected for positions deduced from reductions using USNO A2.0 and GSC 2.2 stars, this could also imply that compared to a nonphotographic catalogue, the main photographic survey catalogues (USNO, GSC) could have the same kind of systematic defects in right ascension. Further investigations based on comparison between photographic and non-photographic catalogs are requiered.

\subsection{USNO B1.0 vs. GSC 2.2}

The similitude of trends in right ascension detected previously in differences between ICRF positions and positions deduced from reductions using GSC 2.2 stars is better verified by the direct comparison of common stars between GSC 2.2 and B1.0 catalogues averaged for the observed fields. The average results are shown in Fig. 8. The systematic offsets detected previously in B1.0 in right ascension and declination are clearly present in Fig. 8, but no systematic trend in right
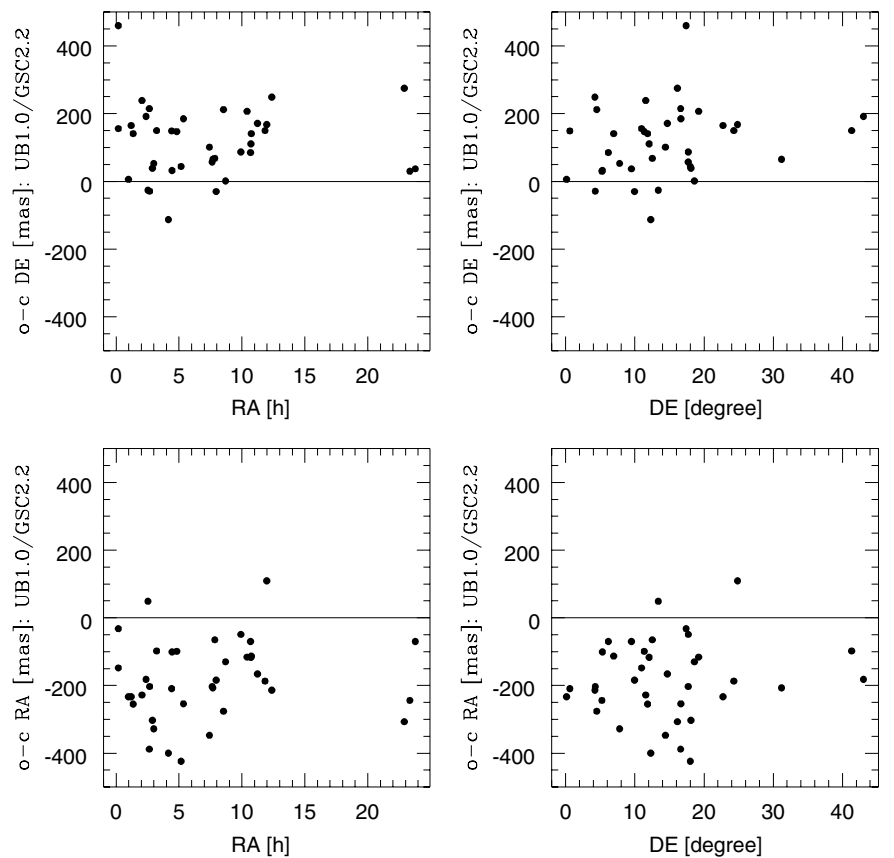

Fig. 8. Mean differences between the USNO B1.0 and GSC 2.2 positions for common stars averaged over the 38 observed fields.

ascension could be detected. The GSC 2.2 being closer to ICRF than the USNO catalogues, this result implies that systematic 
offsets are clearly present in the B1.0 catalogue, but also that the systematic trend in right ascension is common for the B1.0 and the GSC 2.2 catalogue.

\section{Conclusion}

The USNO B1.0 catalogue is unique given the number of sources, evenness of density distribution, and magnitude deepness, among existing catalogues. In this paper a representative portion of one fifth of the northern sky is studied. The quality of positions is assessed by direct catalogue comparison, and through the astrometry for the positions of optical counterparts of 38 ICRF sources. The sources are distributed in intervals from 23 to $13 \mathrm{~h}$ in right ascension and from 0 to +25 degrees in declination. There is one source at 43 degrees in declination. The internal accuracy is found to be 180 mas in right ascension and declination, from the postfit distribution of the residuals to the astrometric reduction. This is comparable to the GSC 2.2 quality, and represents a significant improvement relative to the USNO A2.0 catalogue.

Overall the northern sky, systematic departures from the ICRF are determined by direct comparison of positions at -69 mas in right ascension and +133 mas in declination. The main zonal errors are in the right ascension. Two different groups can be seen, before and after $6^{\mathrm{h}}$. The zonal errors can be compared on the scale of the examined sky region by a linear adjustment of a significant angular coefficient.

The same features are seen from the comparisons based on the ICRF coordinates, on the astrometric reduction of the observations, and on M 2000 common star coordinates. Therefore the range from the 12th mag to the faintest end of the B1.0 catalogue is covered revealing no important magnitude equation.

These same comparisons ensure that no bias was introduced by the present observations and data reduction. On the contrary, the same kind of features found on the GSC 2.2 and A2.0 catalogues imply a cause related to the astrographic plates that furnish the images for the three catalogues.

Acknowledgements. The work described in this paper was possible thanks to the Observatoire de Paris. The observations have been made possible thanks to the OHP staff, the CNRS, the Institut des sciences de l'Univers/CNES and the Observatoire de Paris

\section{References}

Alard, C. 2002, private communication

Assafin, M., Andrei, A. H., Vieira Martins, R., et al. 2001, AJ, 552, 380

ECLIPSE 4.2 2002, http://www.eso.org/projects/aot/eclipse/

Fienga, A. 1999, Ph.D. Thesis., Observatoire de Paris

Fienga, A., \& Andrei, A. H. 2002, A\&A, 393, 331

Fienga, A., Arlot, J.-E., Baron, N., et al. 2002, A\&A, 391, 767

Monet, D., Bird, A., Canzian, B., et al. 1998, The USNO-A2.0 Catalog (Washington DC: U.S. Naval Observatory)

Monet, D. G., Levine, S. E., Canzian, B., et al. 2003, AJ, 125, 984

Rapaport, M., Le Campion, J.-F., Soubiran, C., et al. 2001, A\&A, 376, 325

STScI 2001, http://www-gsss.stsci.edu/gsc/gsc2/GSC2home.htm

Stoughton, C., Lupton, R. H., Bernardi, M., Blanton, M. R., \& Burles, S. 2002, AJ, 123, 485 